Acta Crystallographica Section E

Structure Reports

Online

ISSN 1600-5368

\section{3-(4-Chlorophenylsulfonyl)-2,5-dimethyl- 1-benzofuran}

\section{Hong Dae Choi, ${ }^{a}$ Pil Ja Seo, ${ }^{a}$ Byeng Wha Son ${ }^{b}$ and Uk $\operatorname{Lee}^{\mathrm{b} *}$}

aDepartment of Chemistry, Dongeui University, San 24 Kaya-dong Busanjin-gu, Busan 614-714, Republic of Korea, and ${ }^{\mathbf{b}}$ Department of Chemistry, Pukyong National University, 599-1 Daeyeon 3-dong, Nam-gu, Busan 608-737, Republic of Korea

Correspondence e-mail: uklee@pknu.ac.kr

Received 27 October 2010; accepted 28 October 2010

Key indicators: single-crystal X-ray study; $T=177 \mathrm{~K}$; mean $\sigma(\mathrm{C}-\mathrm{C})=0.003 \AA$; $R$ factor $=0.040 ; w R$ factor $=0.107 ;$ data-to-parameter ratio $=17.7$.

In the title compound, $\mathrm{C}_{16} \mathrm{H}_{13} \mathrm{ClO}_{3} \mathrm{~S}$, the 4-chlorophenyl ring makes a dihedral angle of $79.96(5)^{\circ}$ with the mean plane of the benzofuran fragment. In the crystal, molecules are linked through weak intermolecular $\mathrm{C}-\mathrm{H} \cdots \mathrm{O}$ and $\mathrm{C}-\mathrm{H} \cdots \pi$ interactions.

\section{Related literature}

For the biological activity of benzofuran compounds, see: Aslam et al. (2006); Galal et al. (2009); Khan et al. (2005). For natural products with benzofuran rings, see: Akgul \& Anil (2003); Soekamto et al. (2003). For our previous structural studies of related 3-(4-chlorophenylsulfinyl)-2,5-dimethyl-1benzofuran derivatives, see: Choi et al. $(2010 a, b)$.

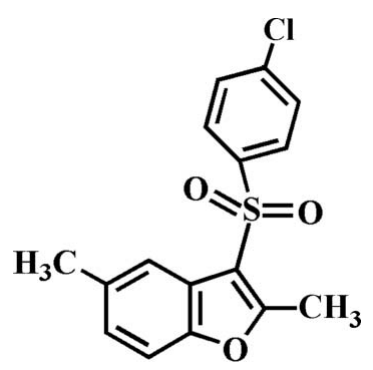

\section{Experimental}

Crystal data

$\mathrm{C}_{16} \mathrm{H}_{13} \mathrm{ClO}_{3} \mathrm{~S}$

$M_{r}=320.77$

Monoclinic, $P 2_{1} / c$ $\beta=105.363(1)^{\circ}$

$V=1486.82(5) \AA^{3}$

$Z=4$

Mo $K \alpha$ radiation

Data collection

Bruker SMART APEXII CCD diffractometer

Absorption correction: multi-scan (SADABS; Bruker, 2009)

$T_{\min }=0.867, T_{\max }=0.962$

Refinement

$R\left[F^{2}>2 \sigma\left(F^{2}\right)\right]=0.040$

$w R\left(F^{2}\right)=0.107$

$S=1.06$

3398 reflections

Table 1

Hydrogen-bond geometry $\left(\AA,{ }^{\circ}\right)$.

$$
\begin{aligned}
& \mu=0.40 \mathrm{~mm}^{-1} \\
& T=177 \mathrm{~K} \\
& 0.37 \times 0.23 \times 0.10 \mathrm{~mm}
\end{aligned}
$$

13864 measured reflections 3398 independent reflections 2655 reflections with $I>2 \sigma(I)$ $R_{\text {int }}=0.033$

192 parameters

$\mathrm{H}$-atom parameters constrained $\Delta \rho_{\max }=0.29{\mathrm{e} \AA^{-3}}^{-3}$

$\Delta \rho_{\min }=-0.34 \mathrm{e}^{-3}$

$\mathrm{Cg}$ is the centroid of the $\mathrm{C} 2-\mathrm{C} 7$ benzene ring.

\begin{tabular}{lllll}
\hline$D-\mathrm{H} \cdots A$ & $D-\mathrm{H}$ & $\mathrm{H} \cdots A$ & $D \cdots A$ & $D-\mathrm{H} \cdots A$ \\
\hline $\mathrm{C} 6-\mathrm{H} 6 \cdots \mathrm{O} 1^{\mathrm{i}}$ & 0.95 & 2.56 & $3.495(2)$ & 167 \\
$\mathrm{C} 10-\mathrm{H} 10 A \cdots \mathrm{O} 2^{\mathrm{ii}}$ & 0.98 & 2.43 & $3.279(2)$ & 145 \\
$\mathrm{C} 13-\mathrm{H} 13 \cdots C g^{\mathrm{iii}}$ & 0.95 & 2.74 & $3.411(2)$ & 128 \\
\hline
\end{tabular}

Symmetry codes: (i) $-x,-y+1,-z+2$; (ii) $x,-y+\frac{3}{2}, z+\frac{1}{2}$; (iii) $x,-y+\frac{3}{2}, z-\frac{1}{2}$.

Data collection: APEX2 (Bruker, 2009); cell refinement: SAINT (Bruker, 2009); data reduction: $S A I N T$; $\operatorname{program}(\mathrm{s})$ used to solve structure: SHELXS97 (Sheldrick, 2008); program(s) used to refine structure: SHELXL97 (Sheldrick, 2008); molecular graphics: ORTEP-3 (Farrugia, 1997) and DIAMOND (Brandenburg, 1998); software used to prepare material for publication: SHELXL97.

This work was supported by Blue-Bio Industry RIC at Dongeui University as an RIC programme under the Ministry of Knowledge Economy and Busan city.

Supplementary data and figures for this paper are available from the IUCr electronic archives (Reference: NG5054).

\title{
References
}

Akgul, Y. Y. \& Anil, H. (2003). Phytochemistry, 63, 939-943.

Aslam, S. N., Stevenson, P. C., Phythian, S. J., Veitch, N. C. \& Hall, D. R. (2006). Tetrahedron, 62, 4214-4226.

Brandenburg, K. (1998). DIAMOND. Crystal Impact GbR, Bonn, Germany. Bruker (2009). APEX2, SADABS and SAINT. Bruker AXS Inc., Madison, Wisconsin, USA.

Choi, H. D., Seo, P. J., Son, B. W. \& Lee, U. (2010a). Acta Cryst. E66, o2325.

Choi, H. D., Seo, P. J., Son, B. W. \& Lee, U. (2010b). Acta Cryst. E66, o2551.

Farrugia, L. J. (1997). J. Appl. Cryst. 30, 565.

Galal, S. A., Abd El-All, A. S., Abdallah, M. M. \& El-Diwani, H. I. (2009). Bioorg. Med. Chem. Lett. 19, 2420-2428.

Khan, M. W., Alam, M. J., Rashid, M. A. \& Chowdhury, R. (2005). Bioorg. Med. Chem. 13, 4796-4805.

Sheldrick, G. M. (2008). Acta Cryst. A64, 112-122.

Soekamto, N. H., Achmad, S. A., Ghisalberti, E. L., Hakim, E. H. \& Syah, Y. M. (2003). Phytochemistry, 64, 831-834. 


\title{
supporting information
}

Acta Cryst. (2010). E66, o3065 [https://doi.org/10.1107/S1600536810044223]

\section{3-(4-Chlorophenylsulfonyl)-2,5-dimethyl-1-benzofuran}

\author{
Hong Dae Choi, Pil Ja Seo, Byeng Wha Son and Uk Lee
}

\section{S1. Comment}

A series of benzofuran ring system has received much attention in view of their particular pharmacological properties such as antifungal, antimicrobial, antitumor and antiviral activities (Aslam et al., 2006; Galal et al., 2009; Khan et al., 2005). These compounds widely occur in nature (Akgul \& Anil, 2003; Soekamto et al., 2003). As a part of our study of the substituent effect on the solid state structures of 3-(4-chlorophenylsulfinyl)-2,5-dimethyl-1-benzofuran analogues (Choi et al., 2010a,b), we report herein on the crystal structure of the title compound.

In the title molecule (Fig. 1), the benzofuran unit is essentially planar, with a mean deviation of 0.003 (1) $\AA$ from the least-squares plane defined by the nine constituent atoms. The 4-chlorophenyl ring makes a dihedral angle of $79.96(5)^{\circ}$ with the mean plane of the benzofuran ring. The crystal packing (Fig. 2) is stabilized by weak intermolecular $\mathrm{C}-\mathrm{H} \cdots \mathrm{O}$ hydrogen bonds: the first one between a benzene $\mathrm{H}$ atom and the furan $\mathrm{O}$ atom (Table 1, $\mathrm{C} 6-\mathrm{H} 6 \cdots \mathrm{O} 1^{\mathrm{i}}$ ), and the second one between a methyl $\mathrm{H}$ atom and the oxygen of the $\mathrm{O}=\mathrm{S}=\mathrm{O}$ unit (Table $1, \mathrm{C} 10-\mathrm{H} 10 \mathrm{~A} \cdots \mathrm{O} 2^{\mathrm{ii}}$ ). The crystal packing (Fig. 2 ) is further stabilized by an intermolecular $\mathrm{C}-\mathrm{H} \cdots \pi$ interaction between the 4-chlorophenyl $\mathrm{H}$ atom and the benzene ring (Table 1; $\mathrm{C} 13-\mathrm{H} 13 \cdots \mathrm{Cg}{ }^{\mathrm{iii}}$, $\mathrm{Cg}$ is the centroid of the $\mathrm{C} 2-\mathrm{C} 7$ benzene ring).

\section{S2. Experimental}

77\% 3-chloroperoxybenzoic acid (269 mg, $1.2 \mathrm{mmol}$ ) was added in small portions to a stirred solution of 3-(4-chlorophenylsulfanyl)-2,5-dimethyl-1-benzofuran $(346 \mathrm{mg}, 1.2 \mathrm{mmol})$ in dichloromethane $(40 \mathrm{~mL})$ at $273 \mathrm{~K}$. After being stirred at room temperature for $6 \mathrm{~h}$, the mixture was washed with saturated sodium bicarbonate solution and the organic layer was separated, dried over magnesium sulfate, filtered and concentrated at reduced pressure. The residue was purified by column chromatography (benzene) to afford the title compound as a colorless solid [yield 81\%, m.p. 428-429 K; $R_{\mathrm{f}}=$ 0.59 (benzene)]. Single crystals suitable for X-ray diffraction were prepared by slow evaporation of a solution of the title compound in acetone at room temperature.

\section{S3. Refinement}

All $\mathrm{H}$ atoms were positioned geometrically and refined using a riding model, with $\mathrm{C}-\mathrm{H}=0.95 \AA$ for aryl and $0.98 \AA$ for methyl $\mathrm{H}$ atoms. $U_{\text {iso }}(\mathrm{H})=1.2 U_{\mathrm{eq}}(\mathrm{C})$ for aryl and $1.5 U_{\mathrm{eq}}(\mathrm{C})$ for methyl $\mathrm{H}$ atoms. 


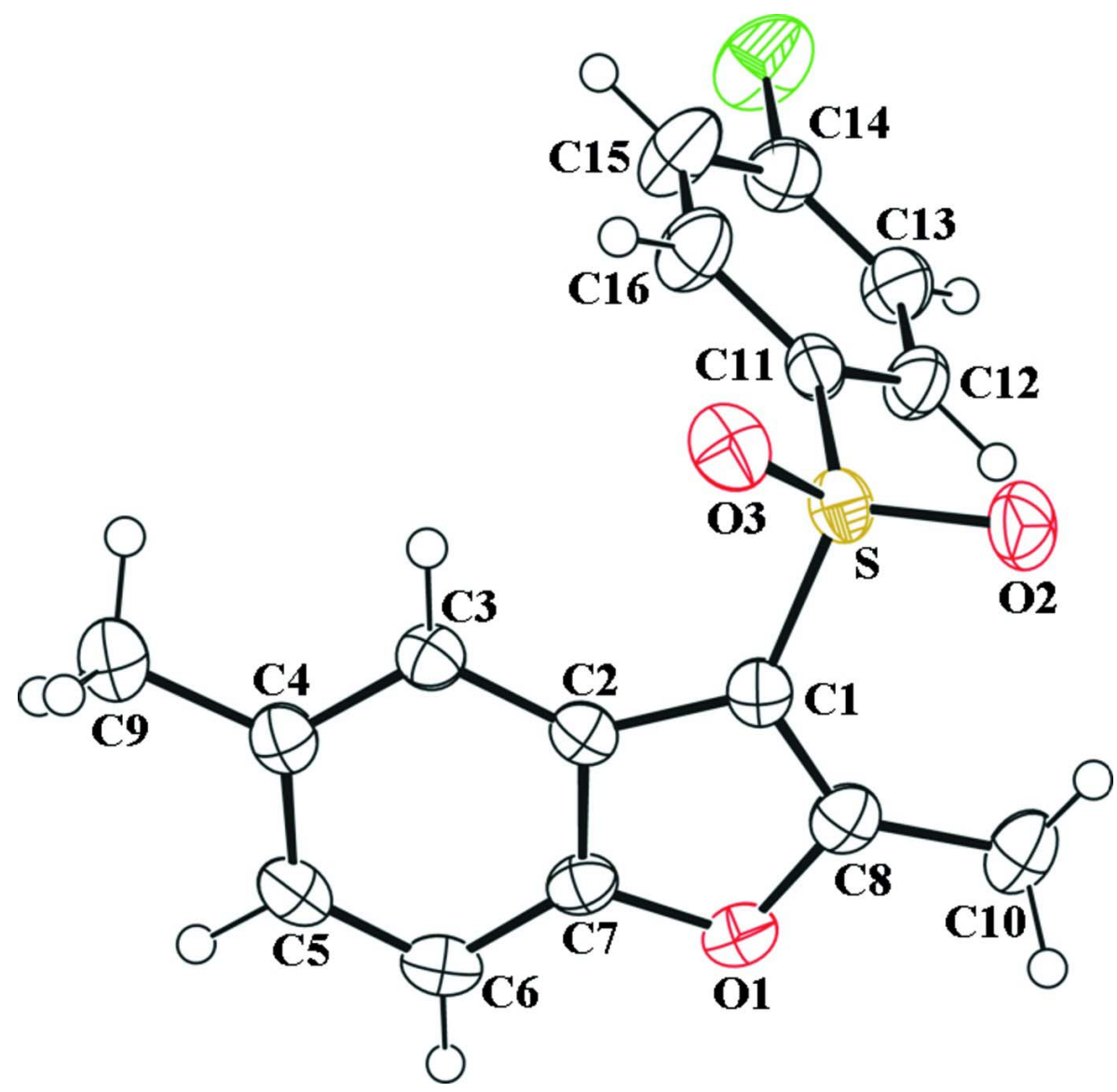

Figure 1

The molecular structure of the title compound with the atom numbering scheme. Displacement ellipsoids are drawn at the $50 \%$ probability level. $\mathrm{H}$ atoms are presented as a small spheres of arbitrary radius. 


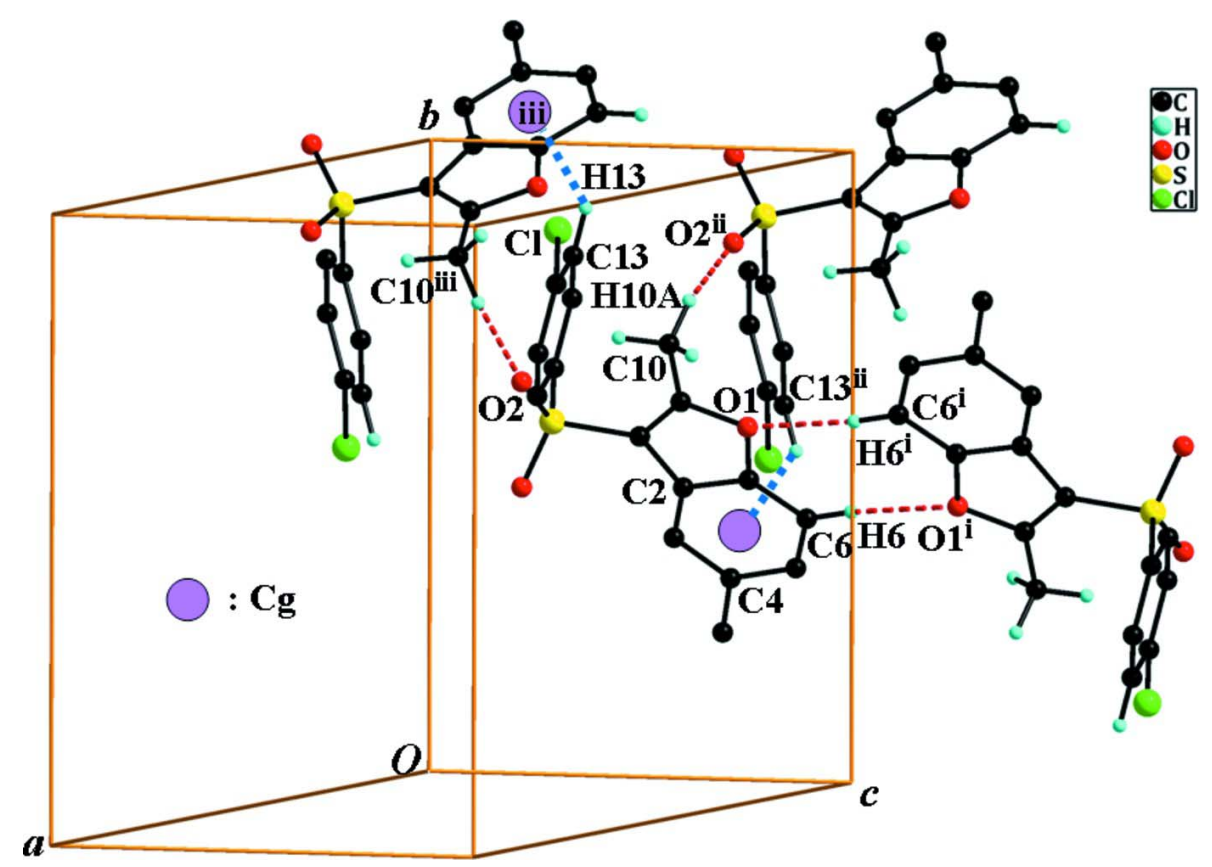

Figure 2

A view of the $\mathrm{C}-\mathrm{H} \cdots \mathrm{O}$ and $\mathrm{C}-\mathrm{H} \cdots \pi$ interactions (dotted lines) in the crystal structure of the title compound. $\mathrm{Cg}$ denotes the centroid of the $\mathrm{C} 2-\mathrm{C} 7$ benzene ring. [Symmetry codes: (i) $-x,-y+1,-z+2$; (ii) $x,-y+3 / 2, z+1 / 2$; (iii) $x,-y+3 / 2, z$ $-1 / 2$.]

\section{3-(4-Chlorophenylsulfonyl)-2,5-dimethyl-1-benzofuran}

\section{Crystal data}

\section{$\mathrm{C}_{16} \mathrm{H}_{13} \mathrm{ClO}_{3} \mathrm{~S}$}

$M_{r}=320.77$

Monoclinic, $P 2_{1} / c$

Hall symbol: -P_2ybc

$a=15.3529$ (3) $\bar{\AA}$

$b=12.1495(2) \AA$

$c=8.2663(2) \AA$

$\beta=105.363(1)^{\circ}$

$V=1486.82(5) \AA^{3}$

$Z=4$

\section{Data collection}

\section{Bruker SMART APEXII CCD}

diffractometer

Radiation source: rotating anode

Graphite multilayer monochromator

Detector resolution: 10.0 pixels $\mathrm{mm}^{-1}$

$\varphi$ and $\omega$ scans

Absorption correction: multi-scan

(SADABS; Bruker, 2009)

$T_{\text {min }}=0.867, T_{\max }=0.962$
$F(000)=664$

$D_{\mathrm{x}}=1.433 \mathrm{Mg} \mathrm{m}^{-3}$

Mo $K \alpha$ radiation, $\lambda=0.71073 \AA$

Cell parameters from 4847 reflections

$\theta=2.8-27.5^{\circ}$

$\mu=0.40 \mathrm{~mm}^{-1}$

$T=177 \mathrm{~K}$

Block, colourless

$0.37 \times 0.23 \times 0.10 \mathrm{~mm}$

13864 measured reflections

3398 independent reflections

2655 reflections with $I>2 \sigma(I)$

$R_{\text {int }}=0.033$

$\theta_{\text {max }}=27.5^{\circ}, \theta_{\min }=1.4^{\circ}$

$h=-19 \rightarrow 19$

$k=-15 \rightarrow 14$

$l=-10 \rightarrow 10$ 


\section{Refinement}

Refinement on $F^{2}$

Least-squares matrix: full

$R\left[F^{2}>2 \sigma\left(F^{2}\right)\right]=0.040$

$w R\left(F^{2}\right)=0.107$

$S=1.06$

3398 reflections

192 parameters

0 restraints

Primary atom site location: structure-invariant direct methods
Secondary atom site location: difference Fourier map

Hydrogen site location: difference Fourier map

$\mathrm{H}$-atom parameters constrained

$w=1 /\left[\sigma^{2}\left(F_{\mathrm{o}}^{2}\right)+(0.0506 P)^{2}+0.4243 P\right]$

where $P=\left(F_{\mathrm{o}}{ }^{2}+2 F_{\mathrm{c}}{ }^{2}\right) / 3$

$(\Delta / \sigma)_{\max }<0.001$

$\Delta \rho_{\max }=0.29 \mathrm{e} \AA^{-3}$

$\Delta \rho_{\min }=-0.34$ e $\AA^{-3}$

Special details

Geometry. All esds (except the esd in the dihedral angle between two 1.s. planes) are estimated using the full covariance matrix. The cell esds are taken into account individually in the estimation of esds in distances, angles and torsion angles; correlations between esds in cell parameters are only used when they are defined by crystal symmetry. An approximate (isotropic) treatment of cell esds is used for estimating esds involving l.s. planes.

Refinement. Refinement of $\mathrm{F}^{2}$ against ALL reflections. The weighted R-factor $\mathrm{wR}$ and goodness of fit $\mathrm{S}$ are based on $\mathrm{F}^{2}$, conventional R-factors $R$ are based on $F$, with $F$ set to zero for negative $F^{2}$. The threshold expression of $\mathrm{F}^{2}>2 \operatorname{sigma}\left(\mathrm{F}^{2}\right)$ is used only for calculating R-factors(gt) etc. and is not relevant to the choice of reflections for refinement. R-factors based on $\mathrm{F}^{2}$ are statistically about twice as large as those based on F, and R- factors based on ALL data will be even larger.

Fractional atomic coordinates and isotropic or equivalent isotropic displacement parameters $\left(\AA^{2}\right)$

\begin{tabular}{lllll}
\hline & $x$ & $y$ & $z$ & $U_{\text {iso }} / U_{\text {eq }}$ \\
\hline Cl & $0.48342(4)$ & $0.92660(5)$ & $0.73305(10)$ & $0.0705(2)$ \\
S & $0.17974(3)$ & $0.58289(4)$ & $0.44925(5)$ & $0.03028(14)$ \\
O1 & $0.04560(8)$ & $0.56425(10)$ & $0.79164(16)$ & $0.0336(3)$ \\
O2 & $0.11294(8)$ & $0.63535(12)$ & $0.31700(15)$ & $0.0417(3)$ \\
O3 & $0.22166(9)$ & $0.48321(11)$ & $0.41515(15)$ & $0.0384(3)$ \\
C1 & $0.13384(11)$ & $0.55688(14)$ & $0.6156(2)$ & $0.0282(4)$ \\
C2 & $0.17212(10)$ & $0.48293(14)$ & $0.75339(19)$ & $0.0265(4)$ \\
C3 & $0.24701(11)$ & $0.41325(14)$ & $0.7986(2)$ & $0.0286(4)$ \\
H3 & 0.2872 & 0.4070 & 0.7293 & $0.034^{*}$ \\
C4 & $0.26171(11)$ & $0.35337(15)$ & $0.9461(2)$ & $0.0305(4)$ \\
C5 & $0.20127(12)$ & $0.36427(16)$ & $1.0465(2)$ & $0.0336(4)$ \\
H5 & 0.2120 & 0.3226 & 1.1471 & $0.040^{*}$ \\
C6 & $0.12720(12)$ & $0.43281(15)$ & $1.0054(2)$ & $0.0346(4)$ \\
H6 & 0.0871 & 0.4400 & 1.0748 & $0.041^{*}$ \\
C7 & $0.11476(11)$ & $0.49046(15)$ & $0.8570(2)$ & $0.0287(4)$ \\
C8 & $0.05880(11)$ & $0.60332(15)$ & $0.6451(2)$ & $0.0312(4)$ \\
C9 & $0.34169(13)$ & $0.27807(18)$ & $0.9993(2)$ & $0.0426(5)$ \\
H9A & 0.3251 & 0.2045 & 0.9527 & $0.064^{*}$ \\
H9B & 0.3608 & 0.2738 & 1.1220 & $0.064^{*}$ \\
H9C & 0.3914 & 0.3068 & 0.9579 & $0.064^{*}$ \\
C10 & $-0.00891(12)$ & $0.68520(16)$ & $0.5565(3)$ & $0.0403(5)$ \\
H10A & 0.0030 & 0.7562 & 0.6145 & $0.060^{*}$ \\
H10B & -0.0696 & 0.6598 & 0.5558 & $0.060^{*}$ \\
H10C & -0.0048 & 0.6936 & 0.4409 & $0.060^{*}$ \\
C11 & $0.26643(11)$ & $0.67929(14)$ & $0.5303(2)$ & $0.0285(4)$ \\
& & & &
\end{tabular}




$\begin{array}{lllll}\text { C12 } & 0.24350(12) & 0.78759(16) & 0.5522(2) & 0.0372(4) \\ \text { H12 } & 0.1818 & 0.8092 & 0.5243 & 0.045^{*} \\ \text { C13 } & 0.31025(13) & 0.86365(16) & 0.6145(3) & 0.0409(5) \\ \text { H13 } & 0.2954 & 0.9381 & 0.6301 & 0.049^{*} \\ \text { C14 } & 0.39885(13) & 0.83017(17) & 0.6538(3) & 0.0418(5) \\ \text { C15 } & 0.42284(13) & 0.72285(18) & 0.6332(3) & 0.0527(6) \\ \text { H15 } & 0.4846 & 0.7016 & 0.6616 & 0.063^{*} \\ \text { C16 } & 0.35555(12) & 0.64652(17) & 0.5705(3) & 0.0435(5) \\ \text { H16 } & 0.3706 & 0.5721 & 0.5552 & 0.052^{*}\end{array}$

Atomic displacement parameters $\left(\AA^{2}\right)$

\begin{tabular}{lllllll}
\hline & $U^{11}$ & $U^{22}$ & $U^{33}$ & $U^{12}$ & $U^{13}$ & $U^{23}$ \\
\hline $\mathrm{C} 1$ & $0.0463(3)$ & $0.0523(4)$ & $0.1062(6)$ & $-0.0155(3)$ & $0.0085(3)$ & $-0.0142(3)$ \\
$\mathrm{S}$ & $0.0351(2)$ & $0.0300(3)$ & $0.0243(2)$ & $0.00103(18)$ & $0.00535(17)$ & $0.00088(17)$ \\
$\mathrm{O} 1$ & $0.0275(6)$ & $0.0353(7)$ & $0.0396(7)$ & $-0.0011(5)$ & $0.0114(5)$ & $-0.0041(6)$ \\
$\mathrm{O} 2$ & $0.0411(7)$ & $0.0475(9)$ & $0.0302(7)$ & $0.0000(6)$ & $-0.0013(5)$ & $0.0064(6)$ \\
$\mathrm{O} 3$ & $0.0524(8)$ & $0.0333(7)$ & $0.0313(6)$ & $0.0023(6)$ & $0.0143(6)$ & $-0.0041(6)$ \\
$\mathrm{C} 1$ & $0.0294(8)$ & $0.0253(9)$ & $0.0285(8)$ & $-0.0018(7)$ & $0.0050(7)$ & $-0.0015(7)$ \\
$\mathrm{C} 2$ & $0.0294(8)$ & $0.0247(9)$ & $0.0251(8)$ & $-0.0053(7)$ & $0.0066(6)$ & $-0.0032(7)$ \\
$\mathrm{C} 3$ & $0.0312(8)$ & $0.0280(9)$ & $0.0281(8)$ & $0.0004(7)$ & $0.0105(7)$ & $-0.0024(7)$ \\
$\mathrm{C} 4$ & $0.0328(9)$ & $0.0276(9)$ & $0.0294(8)$ & $-0.0032(7)$ & $0.0050(7)$ & $-0.0005(7)$ \\
$\mathrm{C} 5$ & $0.0402(10)$ & $0.0331(10)$ & $0.0276(8)$ & $-0.0092(8)$ & $0.0090(7)$ & $0.0012(8)$ \\
$\mathrm{C} 6$ & $0.0358(9)$ & $0.0377(11)$ & $0.0340(9)$ & $-0.0083(8)$ & $0.0160(8)$ & $-0.0043(8)$ \\
$\mathrm{C} 7$ & $0.0254(8)$ & $0.0279(9)$ & $0.0333(9)$ & $-0.0036(7)$ & $0.0086(7)$ & $-0.0050(7)$ \\
$\mathrm{C} 8$ & $0.0288(8)$ & $0.0283(9)$ & $0.0338(9)$ & $-0.0042(7)$ & $0.0039(7)$ & $-0.0053(8)$ \\
$\mathrm{C} 9$ & $0.0444(11)$ & $0.0420(12)$ & $0.0403(10)$ & $0.0056(9)$ & $0.0092(9)$ & $0.0095(9)$ \\
C10 & $0.0325(9)$ & $0.0334(11)$ & $0.0498(11)$ & $0.0038(8)$ & $0.0016(8)$ & $-0.0051(9)$ \\
C11 & $0.0325(8)$ & $0.0277(9)$ & $0.0254(8)$ & $0.0027(7)$ & $0.0076(7)$ & $0.0041(7)$ \\
C12 & $0.0316(9)$ & $0.0315(10)$ & $0.0471(11)$ & $0.0065(8)$ & $0.0082(8)$ & $0.0035(9)$ \\
C13 & $0.0429(10)$ & $0.0281(10)$ & $0.0522(12)$ & $0.0023(8)$ & $0.0134(9)$ & $0.0013(9)$ \\
C14 & $0.0361(10)$ & $0.0380(11)$ & $0.0490(11)$ & $-0.0068(8)$ & $0.0074(8)$ & $-0.0018(9)$ \\
C15 & $0.0299(10)$ & $0.0440(13)$ & $0.0790(16)$ & $0.0052(9)$ & $0.0055(10)$ & $-0.0068(12)$ \\
C16 & $0.0358(10)$ & $0.0337(11)$ & $0.0591(13)$ & $0.0073(8)$ & $0.0092(9)$ & $-0.0028(10)$ \\
& & & & & & \\
\hline
\end{tabular}

Geometric parameters $\left(A,{ }^{\circ}\right)$

\begin{tabular}{llll}
\hline $\mathrm{Cl}-\mathrm{C} 14$ & $1.743(2)$ & $\mathrm{C} 6-\mathrm{H} 6$ & 0.9500 \\
$\mathrm{~S}-\mathrm{O} 3$ & $1.4339(14)$ & $\mathrm{C} 8-\mathrm{C} 10$ & $1.484(2)$ \\
$\mathrm{S}-\mathrm{O} 2$ & $1.4350(13)$ & $\mathrm{C} 9-\mathrm{H} 9 \mathrm{~A}$ & 0.9800 \\
$\mathrm{~S}-\mathrm{C} 1$ & $1.7319(17)$ & $\mathrm{C} 9-\mathrm{H} 9 \mathrm{~B}$ & 0.9800 \\
$\mathrm{~S}-\mathrm{C} 11$ & $1.7664(18)$ & $\mathrm{C} 9-\mathrm{H} 9 \mathrm{C}$ & 0.9800 \\
$\mathrm{O} 1-\mathrm{C} 8$ & $1.366(2)$ & $\mathrm{C} 10-\mathrm{H} 10 \mathrm{~A}$ & 0.9800 \\
$\mathrm{O} 1-\mathrm{C} 7$ & $1.385(2)$ & $\mathrm{C} 10-\mathrm{H} 10 \mathrm{~B}$ & 0.9800 \\
$\mathrm{C} 1-\mathrm{C} 8$ & $1.362(2)$ & $\mathrm{C} 10-\mathrm{H} 10 \mathrm{C}$ & 0.9800 \\
$\mathrm{C} 1-\mathrm{C} 2$ & $1.448(2)$ & $\mathrm{C} 11-\mathrm{C} 16$ & $1.378(2)$ \\
$\mathrm{C} 2-\mathrm{C} 7$ & $1.384(2)$ & $\mathrm{C} 11-\mathrm{C} 12$ & $1.386(3)$ \\
$\mathrm{C} 2-\mathrm{C} 3$ & $1.397(2)$ & $\mathrm{C} 12-\mathrm{C} 13$ & $1.375(3)$
\end{tabular}




\begin{tabular}{|c|c|c|c|}
\hline $\mathrm{C} 3-\mathrm{C} 4$ & $1.386(2)$ & $\mathrm{C} 12-\mathrm{H} 12$ & 0.9500 \\
\hline $\mathrm{C} 3-\mathrm{H} 3$ & 0.9500 & $\mathrm{C} 13-\mathrm{C} 14$ & $1.374(3)$ \\
\hline $\mathrm{C} 4-\mathrm{C} 5$ & $1.406(2)$ & $\mathrm{C} 13-\mathrm{H} 13$ & 0.9500 \\
\hline $\mathrm{C} 4-\mathrm{C} 9$ & $1.501(2)$ & $\mathrm{C} 14-\mathrm{C} 15$ & $1.378(3)$ \\
\hline $\mathrm{C} 5-\mathrm{C} 6$ & $1.377(3)$ & $\mathrm{C} 15-\mathrm{C} 16$ & $1.383(3)$ \\
\hline $\mathrm{C} 5-\mathrm{H} 5$ & 0.9500 & $\mathrm{C} 15-\mathrm{H} 15$ & 0.9500 \\
\hline $\mathrm{C} 6-\mathrm{C} 7$ & $1.381(3)$ & $\mathrm{C} 16-\mathrm{H} 16$ & 0.9500 \\
\hline $\mathrm{O} 3-\mathrm{S}-\mathrm{O} 2$ & $119.53(8)$ & $\mathrm{C} 4-\mathrm{C} 9-\mathrm{H} 9 \mathrm{~A}$ & 109.5 \\
\hline $\mathrm{O} 3-\mathrm{S}-\mathrm{C} 1$ & $107.47(8)$ & $\mathrm{C} 4-\mathrm{C} 9-\mathrm{H} 9 \mathrm{~B}$ & 109.5 \\
\hline $\mathrm{O} 2-\mathrm{S}-\mathrm{C} 1$ & $109.07(8)$ & $\mathrm{H} 9 \mathrm{~A}-\mathrm{C} 9-\mathrm{H} 9 \mathrm{~B}$ & 109.5 \\
\hline $\mathrm{O} 3-\mathrm{S}-\mathrm{C} 11$ & $107.60(8)$ & $\mathrm{C} 4-\mathrm{C} 9-\mathrm{H} 9 \mathrm{C}$ & 109.5 \\
\hline $\mathrm{O} 2-\mathrm{S}-\mathrm{C} 11$ & $108.01(8)$ & $\mathrm{H} 9 \mathrm{~A}-\mathrm{C} 9-\mathrm{H} 9 \mathrm{C}$ & 109.5 \\
\hline $\mathrm{C} 1-\mathrm{S}-\mathrm{C} 11$ & $104.12(8)$ & $\mathrm{H} 9 \mathrm{~B}-\mathrm{C} 9-\mathrm{H} 9 \mathrm{C}$ & 109.5 \\
\hline $\mathrm{C} 8-\mathrm{O} 1-\mathrm{C} 7$ & $107.00(13)$ & $\mathrm{C} 8-\mathrm{C} 10-\mathrm{H} 10 \mathrm{~A}$ & 109.5 \\
\hline $\mathrm{C} 8-\mathrm{C} 1-\mathrm{C} 2$ & $107.60(15)$ & $\mathrm{C} 8-\mathrm{C} 10-\mathrm{H} 10 \mathrm{~B}$ & 109.5 \\
\hline $\mathrm{C} 8-\mathrm{C} 1-\mathrm{S}$ & $127.45(14)$ & $\mathrm{H} 10 \mathrm{~A}-\mathrm{C} 10-\mathrm{H} 10 \mathrm{~B}$ & 109.5 \\
\hline $\mathrm{C} 2-\mathrm{C} 1-\mathrm{S}$ & $124.91(13)$ & $\mathrm{C} 8-\mathrm{C} 10-\mathrm{H} 10 \mathrm{C}$ & 109.5 \\
\hline $\mathrm{C} 7-\mathrm{C} 2-\mathrm{C} 3$ & $119.21(15)$ & $\mathrm{H} 10 \mathrm{~A}-\mathrm{C} 10-\mathrm{H} 10 \mathrm{C}$ & 109.5 \\
\hline $\mathrm{C} 7-\mathrm{C} 2-\mathrm{C} 1$ & $104.70(15)$ & $\mathrm{H} 10 \mathrm{~B}-\mathrm{C} 10-\mathrm{H} 10 \mathrm{C}$ & 109.5 \\
\hline $\mathrm{C} 3-\mathrm{C} 2-\mathrm{C} 1$ & $136.09(15)$ & $\mathrm{C} 16-\mathrm{C} 11-\mathrm{C} 12$ & $120.86(17)$ \\
\hline $\mathrm{C} 4-\mathrm{C} 3-\mathrm{C} 2$ & $118.96(15)$ & $\mathrm{C} 16-\mathrm{C} 11-\mathrm{S}$ & $119.99(14)$ \\
\hline $\mathrm{C} 4-\mathrm{C} 3-\mathrm{H} 3$ & 120.5 & $\mathrm{C} 12-\mathrm{C} 11-\mathrm{S}$ & $119.15(13)$ \\
\hline $\mathrm{C} 2-\mathrm{C} 3-\mathrm{H} 3$ & 120.5 & $\mathrm{C} 13-\mathrm{C} 12-\mathrm{C} 11$ & $119.80(17)$ \\
\hline $\mathrm{C} 3-\mathrm{C} 4-\mathrm{C} 5$ & $119.40(16)$ & $\mathrm{C} 13-\mathrm{C} 12-\mathrm{H} 12$ & 120.1 \\
\hline $\mathrm{C} 3-\mathrm{C} 4-\mathrm{C} 9$ & $120.51(16)$ & $\mathrm{C} 11-\mathrm{C} 12-\mathrm{H} 12$ & 120.1 \\
\hline $\mathrm{C} 5-\mathrm{C} 4-\mathrm{C} 9$ & $120.09(16)$ & $\mathrm{C} 14-\mathrm{C} 13-\mathrm{C} 12$ & $118.87(18)$ \\
\hline $\mathrm{C} 6-\mathrm{C} 5-\mathrm{C} 4$ & $122.84(16)$ & $\mathrm{C} 14-\mathrm{C} 13-\mathrm{H} 13$ & 120.6 \\
\hline $\mathrm{C} 6-\mathrm{C} 5-\mathrm{H} 5$ & 118.6 & $\mathrm{C} 12-\mathrm{C} 13-\mathrm{H} 13$ & 120.6 \\
\hline $\mathrm{C} 4-\mathrm{C} 5-\mathrm{H} 5$ & 118.6 & $\mathrm{C} 13-\mathrm{C} 14-\mathrm{C} 15$ & $122.10(18)$ \\
\hline $\mathrm{C} 5-\mathrm{C} 6-\mathrm{C} 7$ & $115.89(16)$ & $\mathrm{C} 13-\mathrm{C} 14-\mathrm{Cl}$ & $118.84(16)$ \\
\hline $\mathrm{C} 5-\mathrm{C} 6-\mathrm{H} 6$ & 122.1 & $\mathrm{C} 15-\mathrm{C} 14-\mathrm{Cl}$ & $119.06(15)$ \\
\hline $\mathrm{C} 7-\mathrm{C} 6-\mathrm{H} 6$ & 122.1 & $\mathrm{C} 14-\mathrm{C} 15-\mathrm{C} 16$ & $118.91(18)$ \\
\hline $\mathrm{C} 6-\mathrm{C} 7-\mathrm{C} 2$ & $123.71(16)$ & $\mathrm{C} 14-\mathrm{C} 15-\mathrm{H} 15$ & 120.5 \\
\hline $\mathrm{C} 6-\mathrm{C} 7-\mathrm{O} 1$ & $125.86(15)$ & $\mathrm{C} 16-\mathrm{C} 15-\mathrm{H} 15$ & 120.5 \\
\hline $\mathrm{C} 2-\mathrm{C} 7-\mathrm{O} 1$ & $110.43(15)$ & $\mathrm{C} 11-\mathrm{C} 16-\mathrm{C} 15$ & $119.46(18)$ \\
\hline $\mathrm{C} 1-\mathrm{C} 8-\mathrm{O} 1$ & $110.27(15)$ & $\mathrm{C} 11-\mathrm{C} 16-\mathrm{H} 16$ & 120.3 \\
\hline $\mathrm{C} 1-\mathrm{C} 8-\mathrm{C} 10$ & $134.82(17)$ & $\mathrm{C} 15-\mathrm{C} 16-\mathrm{H} 16$ & 120.3 \\
\hline $\mathrm{O} 1-\mathrm{C} 8-\mathrm{C} 10$ & $114.91(15)$ & & \\
\hline $\mathrm{O} 3-\mathrm{S}-\mathrm{C} 1-\mathrm{C} 8$ & $-148.80(16)$ & $\mathrm{C} 8-\mathrm{O} 1-\mathrm{C} 7-\mathrm{C} 2$ & $0.37(18)$ \\
\hline $\mathrm{O} 2-\mathrm{S}-\mathrm{C} 1-\mathrm{C} 8$ & $-17.84(19)$ & $\mathrm{C} 2-\mathrm{C} 1-\mathrm{C} 8-\mathrm{O} 1$ & $-0.30(19)$ \\
\hline $\mathrm{C} 11-\mathrm{S}-\mathrm{C} 1-\mathrm{C} 8$ & $97.26(17)$ & $\mathrm{S}-\mathrm{C} 1-\mathrm{C} 8-\mathrm{O} 1$ & $-178.16(12)$ \\
\hline $\mathrm{O} 3-\mathrm{S}-\mathrm{C} 1-\mathrm{C} 2$ & $33.69(16)$ & $\mathrm{C} 2-\mathrm{C} 1-\mathrm{C} 8-\mathrm{C} 10$ & $178.95(18)$ \\
\hline $\mathrm{O} 2-\mathrm{S}-\mathrm{C} 1-\mathrm{C} 2$ & $164.64(14)$ & $\mathrm{S}-\mathrm{C} 1-\mathrm{C} 8-\mathrm{C} 10$ & $1.1(3)$ \\
\hline $\mathrm{C} 11-\mathrm{S}-\mathrm{C} 1-\mathrm{C} 2$ & $-80.26(15)$ & $\mathrm{C} 7-\mathrm{O} 1-\mathrm{C} 8-\mathrm{C} 1$ & $-0.03(18)$ \\
\hline $\mathrm{C} 8-\mathrm{C} 1-\mathrm{C} 2-\mathrm{C} 7$ & $0.51(18)$ & $\mathrm{C} 7-\mathrm{O} 1-\mathrm{C} 8-\mathrm{C} 10$ & $-179.45(14)$ \\
\hline $\mathrm{S}-\mathrm{C} 1-\mathrm{C} 2-\mathrm{C} 7$ & $178.44(13)$ & $\mathrm{O} 3-\mathrm{S}-\mathrm{C} 11-\mathrm{C} 16$ & $-8.62(18)$ \\
\hline
\end{tabular}




$\begin{array}{llll}\mathrm{C} 8-\mathrm{C} 1-\mathrm{C} 2-\mathrm{C} 3 & -179.35(18) & \mathrm{O} 2-\mathrm{S}-\mathrm{C} 11-\mathrm{C} 16 & -138.91(15) \\ \mathrm{S}-\mathrm{C} 1-\mathrm{C} 2-\mathrm{C} 3 & -1.4(3) & \mathrm{C} 1-\mathrm{S}-\mathrm{C} 11-\mathrm{C} 16 & 105.24(16) \\ \mathrm{C} 7-\mathrm{C} 2-\mathrm{C} 3-\mathrm{C} 4 & 0.1(2) & \mathrm{O} 3-\mathrm{S}-\mathrm{C} 11-\mathrm{C} 12 & 171.21(14) \\ \mathrm{C} 1-\mathrm{C} 2-\mathrm{C} 3-\mathrm{C} 4 & 179.94(18) & \mathrm{O} 2-\mathrm{S}-\mathrm{C} 11-\mathrm{C} 12 & -74.93(16) \\ \mathrm{C} 2-\mathrm{C} 3-\mathrm{C} 4-\mathrm{C} 5 & \mathrm{C} 1-\mathrm{S}-\mathrm{C} 11-\mathrm{C} 12 & 0.1(3) \\ \mathrm{C} 2-\mathrm{C} 3-\mathrm{C} 4-\mathrm{C} 9 & \mathrm{C} 16-\mathrm{C} 11-\mathrm{C} 12-\mathrm{C} 13 & -179.71(14) \\ \mathrm{C} 3-\mathrm{C} 4-\mathrm{C} 5-\mathrm{C} 6 & \mathrm{~S}-\mathrm{C} 11-\mathrm{C} 12-\mathrm{C} 13 & 0.0(3) \\ \mathrm{C} 9-\mathrm{C} 4-\mathrm{C} 5-\mathrm{C} 6 & \mathrm{C} 11-\mathrm{C} 12-\mathrm{C} 13-\mathrm{C} 14 & -0.2(3) \\ \mathrm{C} 4-\mathrm{C} 5-\mathrm{C} 6-\mathrm{C} 7 & \mathrm{C} 12-\mathrm{C} 13-\mathrm{C} 14-\mathrm{C} 15 & -179.91(15) \\ \mathrm{C} 5-\mathrm{C} 6-\mathrm{C} 7-\mathrm{C} 2 & \mathrm{C} 12-\mathrm{C} 13-\mathrm{C} 14-\mathrm{C} 1 & 0.2(4) \\ \mathrm{C} 5-\mathrm{C} 6-\mathrm{C} 7-\mathrm{O} 1 & \mathrm{C} 13-\mathrm{C} 14-\mathrm{C} 15-\mathrm{C} 16 & 179.91(18) \\ \mathrm{C} 3-\mathrm{C} 2-\mathrm{C} 7-\mathrm{C} 6 & -0.6(3) & \mathrm{C} 1-\mathrm{C} 14-\mathrm{C} 15-\mathrm{C} 16 & -0.1(3) \\ \mathrm{C} 1-\mathrm{C} 2-\mathrm{C} 7-\mathrm{C} 6 & \mathrm{C} 12-\mathrm{C} 11-\mathrm{C} 16-\mathrm{C} 15 & 179.72(17) \\ \mathrm{C} 3-\mathrm{C} 2-\mathrm{C} 7-\mathrm{O} 1 & -179.53(16) & \mathrm{S}-\mathrm{C} 11-\mathrm{C} 16-\mathrm{C} 15 & 0.0(3) \\ \mathrm{C} 1-\mathrm{C} 2-\mathrm{C} 7-\mathrm{O} 1 & \mathrm{C} 14-\mathrm{C} 15-\mathrm{C} 16-\mathrm{C} 11 & \\ \mathrm{C} 8-\mathrm{O} 1-\mathrm{C} 7-\mathrm{C} 6 & -0.54(18) & & \end{array}$

Hydrogen-bond geometry $\left(A,{ }^{\circ}\right)$

$\mathrm{Cg}$ is the centroid of the $\mathrm{C} 2-\mathrm{C} 7$ benzene ring.

\begin{tabular}{lllll}
\hline$D-\mathrm{H} \cdots A$ & $D-\mathrm{H}$ & $\mathrm{H} \cdots A$ & $D \cdots A$ & $D-\mathrm{H} \cdots A$ \\
\hline $\mathrm{C} 6-\mathrm{H} 6 \cdots \mathrm{O} 1^{\mathrm{i}}$ & 0.95 & 2.56 & $3.495(2)$ & 167 \\
$\mathrm{C} 10-\mathrm{H} 10 A \cdots \mathrm{O} 2^{\mathrm{ii}}$ & 0.98 & 2.43 & $3.279(2)$ & 145 \\
$\mathrm{C} 13-\mathrm{H} 13 \cdots C g^{\mathrm{iii}}$ & 0.95 & 2.74 & $3.411(2)$ & 128
\end{tabular}

Symmetry codes: (i) $-x,-y+1,-z+2$; (ii) $x,-y+3 / 2, z+1 / 2$; (iii) $x,-y+3 / 2, z-1 / 2$. 